University of Nebraska - Lincoln

DigitalCommons@University of Nebraska - Lincoln

Norman R. Simon Papers

Research Papers in Physics and Astronomy

7-10-1994

\title{
OPACITY, METALLICITY, AND CEPHEID PERIOD RATIOS IN THE GALAXY AND MAGELLANIC CLOUDS
}

Norman R. Simon

University of Nebraska - Lincoln, nsimon@unl.edu

Shashi M. Kanbur

University of Nebraska-Lincoln, kanbur@oswego.edu

Follow this and additional works at: https://digitalcommons.unl.edu/physicssimon

Simon, Norman R. and Kanbur, Shashi M., "OPACITY, METALLICITY, AND CEPHEID PERIOD RATIOS IN THE GALAXY AND MAGELLANIC CLOUDS" (1994). Norman R. Simon Papers. 43.

https://digitalcommons.unl.edu/physicssimon/43

This Article is brought to you for free and open access by the Research Papers in Physics and Astronomy at DigitalCommons@University of Nebraska - Lincoln. It has been accepted for inclusion in Norman R. Simon Papers by an authorized administrator of DigitalCommons@University of Nebraska - Lincoln. 


\title{
OPACITY, METALLICITY, AND CEPHEID PERIOD RATIOS IN THE GALAXY AND MAGELLANIC CLOUDS
}

\author{
NORMAN R. SimON AND SHASHI M. KANBUR ${ }^{1}$ \\ Department of Physics and Astronomy, University of Nebraska, Lincoln, NE 68588-0111 \\ Received 1993 August 23; accepted 1994 January 14
}

\begin{abstract}
Linear pulsation calculations are employed to reproduce the bump Cepheid resonance $\left(P_{2} / P_{0}=0.5\right.$ at $P_{0} \cong 10$ days) and to model, individually, the $P_{1} / P_{0}$ period ratios for the dozen known Galactic beat Cepheids. Convection is ignored. The results point to a range of metallicity among the Cepheids, perhaps as large as $0.01 \lesssim Z \lesssim 0.02$, with no evidence for any star exceeding $Z=0.02$. We find masses and luminosities which range from $M \lesssim 4 M_{\odot}, \log L \lesssim 3.0$ at $P_{0} \cong 3$ days to $M \lesssim 6 M_{\odot}, \log L \gtrsim 3.5$ at $P_{0} \cong 10$ days. Similar parameters are indicated for the $P_{0} \cong 10$ days Cepheids in the LMC and SMC, provided that the resonance for these stars occurs at a slightly longer period, $P_{0} \gtrsim 11$ days, as has been suggested in the literature. Our calculations were performed mainly using OPAL opacities, but also with new opacities from the Opacity Project (OP). Only small differences were found between the OPAL results and those from OP. Finally, some suggestions are made for possible future work, including evolution and pulsation calculations, and more precise observations of Cepheids in the Magellanic Clouds.
\end{abstract}

Subject headings: Cepheids - Magellanic Clouds - stars: abundances - stars: interiors - stars: oscillations

\section{INTRODUCTION}

The study of "beat" and "bump" Cepheids offers a unique opportunity to obtain information about stellar masses and luminosities and to constrain the theory of stellar evolution. The beat Cepheids pulsate simultaneously in the fundamental and first-overtone modes, and the two periods $\left(P_{0}\right.$ and $\left.P_{1}\right)$ can be accurately measured. In the case of the bump Cepheids, the fundamental period, $P_{0}$, is measured and a second period (that of the second overtone, $P_{2}$ ) is inferred, based upon the controlling presence of a resonance $\left(P_{2} / P_{0}=0.5\right)$ determining the light curve structure in these stars (Simon \& Schmidt 1976; Kovács \& Buchler 1989).

Historically, pulsation calculations on stellar models evolving through the instability strip have yielded period ratios $\left(P_{1} / P_{0}\right.$ and $P_{2} / P_{0}$ for the beat and bump stars, respectively) much larger than those actually observed, thus giving rise to a discrepancy between pulsation theory and the theory of stellar evolution. At the same time, a controversy has arisen regarding how much overshoot occurs at the boundaries of stellar convective cores (e.g., Chiosi 1990). So far as the Cepheids are concerned, increasing the overshoot yields a higher luminosity at given mass.

Very recently, with the advent of new radiative opacities, the gross period-ratio discrepancy has all but vanished. Moskalik, Buchler, \& Marom (1992) have used the OPAL opacities (Iglesias \& Rogers 1991) to obtain roughly the observed period ratios with evolutionary models in both the beat and bump Cepheid regimes. This finding was reproduced by Kanbur \& Simon (1994), who also obtained a very similar result with yet another set of new opacities, namely those of the Opacity Project (Seaton et al. 1993). On the other hand, neither the Moskalik et al. nor the Kanbur-Simon study was extensive or specific enough to attempt to constrain the evolutionary models in any detail.

In the current work we calculate a large number of linear nonadiabatic (LNA) pulsation models, designed to mimic the

\footnotetext{
${ }^{1}$ Postal address: Department of Physics and Astronomy, University of Glasgow, Glasgow G12 8QQ, Scotland.
}

observed periods of the dozen known beat Cepheids and to reproduce the bump Cepheid resonance. As we shall see, these models yield information on Cepheid mass, luminosity and metallicity, and will be useful in guiding future evolution calculations which seek to reproduce the properties of the beat and bump stars.

\section{THE BUMP CEPHEID RESONANCE}

\subsection{Galactic Cepheids}

It is now accepted that the Hertzsprung progression of light curve shape with period has as its seat an accidental resonance between the fundamental and second overtone modes. Graphic expression of the resonance phenomenon occurs when the light curves of galactic classical Cepheids are Fourier decomposed and certain combinations of the coefficients plotted versus period. The plots all show a sharp feature at about 10 days (e.g., Simon \& Moffett 1985), indicating the resonance center, $P_{2} / P_{0}=0.5$. Because of scatter in these diagrams, the precise location of the resonance is uncertain by perhaps \pm 0.5 day (Moskalik et al. 1992).

We have constructed a series of LNA models aimed at matching the resonance. The results are summarized in Table 1, for assumed resonance centers of 9.5, 10.0, and 10.5 days, and for three different metallicities: $X=0.7, Z=0.01,0.02,0.03$. The models were calculated with the strictly radiative LNA code described by Aikawa \& Simon (1983) and using OPAL opacities which includes effects due to intermediate coupling (Iglesias, Rogers, \& Wilson 1992). Our procedure was as follows. For each temperature, the LNA calculations were controlled by a Newton-Raphson iteration which, starting from an initial guess $(M, \log L)$, continued to calculate models until the condition $P_{2} / P_{0}=0.5$ was satisfied at the assumed resonance center $P_{0}=9.5,10$, or 10.5 days. Table 2 shows calculations made according to an identical procedure but using opacities from the Opacity Project (OP), including effects due to fine structure (Seaton et al. 1993).

We notice the following trends in Tables 1 and 2. The inferred masses and luminosities increase with both the 
TABLE 1

Pulsation Models with $P_{2} / P_{0}=0.5$ For $P_{0}=9.5,10.0$, AND 10.5 DAYS (OPACITY Is OPAL)

\begin{tabular}{|c|c|c|c|c|c|c|}
\hline \multirow[b]{2}{*}{$T_{e}$} & \multicolumn{2}{|c|}{$Z=0.01$} & \multicolumn{2}{|c|}{$Z=0.02$} & \multicolumn{2}{|c|}{$Z=0.03$} \\
\hline & $M$ & $\log L$ & $M$ & $\log L$ & $M$ & $\log L$ \\
\hline \multicolumn{7}{|c|}{$P_{0}=9.5$ days } \\
\hline $5100 \ldots$ & 4.47 & 3.32 & 4.91 & 3.35 & 5.49 & 3.38 \\
\hline $5300 .$. & 4.46 & 3.39 & 5.08 & 3.43 & 5.77 & 3.47 \\
\hline $5500 .$. & 4.47 & 3.45 & 5.22 & 3.50 & 6.08 & 3.55 \\
\hline $5700 \ldots$ & 4.42 & 3.51 & 5.40 & 3.57 & 6.39 & 3.62 \\
\hline $5900 \ldots$ & 4.21 & 3.55 & 5.39 & 3.63 & 6.68 & 3.70 \\
\hline \multicolumn{7}{|c|}{$P_{0}=10.0$ days } \\
\hline $5100 \ldots$ & 4.75 & 3.37 & 5.24 & 3.40 & 5.86 & 3.43 \\
\hline 5300. & 4.75 & 3.44 & 5.48 & 3.48 & 6.19 & 3.52 \\
\hline $5500 .$. & 4.77 & 3.50 & 5.62 & 3.55 & 6.54 & 3.60 \\
\hline $5700 \ldots$ & 4.73 & 3.56 & 5.76 & 3.62 & 6.89 & 3.67 \\
\hline $5900 \ldots$ & 4.48 & 3.60 & 5.81 & 3.68 & 7.24 & 3.75 \\
\hline \multicolumn{7}{|c|}{$P_{0}=10.5$ days } \\
\hline $5100 \ldots$ & 5.02 & 3.41 & 5.58 & 3.45 & 6.28 & 3.48 \\
\hline $5300 \ldots \ldots \ldots$ & 5.05 & 3.48 & 5.79 & 3.52 & 6.63 & 3.56 \\
\hline $5500 \ldots \ldots$ & 5.07 & 3.55 & 6.00 & 3.60 & 7.02 & 3.65 \\
\hline $5700 \ldots$ & 5.04 & 3.61 & 6.19 & 3.67 & 7.42 & 3.72 \\
\hline $5900 \ldots$ & 4.77 & 3.65 & 6.28 & 3.73 & 7.78 & 3.80 \\
\hline
\end{tabular}

assumed metallicity and the assumed period of the resonance center. This agrees with the results of Moskalik et al. (1992). In addition, we see (generally) an increase of inferred mass and luminosity-to-mass ratio with higher assumed temperature. Compared with OPAL (Table 1), the Opacity Project opacities (Table 2) tend to give higher masses and luminosities at each temperature, and this effect increases with $Z$.

What is the correct temperature to assume on the average for an ensemble of galactic classical Cepheids with periods near 10 days? To answer this question we have examined Cepheids with periods between 9 and 11 days from two large observational samples-namely, those of Moffett \& Barnes (1985) and

TABLE 2

Pulsation Models With $P_{2} / P_{0}=0.5$ for $P_{0}=9.5,10.0$, AND 10.5 DAYS (OPACITY Is OP)

\begin{tabular}{|c|c|c|c|c|c|c|}
\hline \multirow[b]{2}{*}{$T_{e}$} & \multicolumn{2}{|c|}{$Z=0.01$} & \multicolumn{2}{|c|}{$Z=0.02$} & \multicolumn{2}{|c|}{$Z=0.03$} \\
\hline & $M$ & $\log L$ & $M$ & $\log L$ & $M$ & $\log L$ \\
\hline \multicolumn{7}{|c|}{$P_{0}=9.5$ days } \\
\hline 5100. & 4.50 & 3.32 & 5.14 & 3.36 & 6.23 & 3.42 \\
\hline $5300 \ldots$ & 4.47 & 3.39 & 5.40 & 3.45 & 6.59 & 3.51 \\
\hline $5500 \ldots$ & 4.48 & 3.45 & 5.69 & 3.53 & 7.18 & 3.60 \\
\hline $5700 \ldots$ & 4.33 & 3.50 & 5.67 & 3.58 & 7.52 & 3.67 \\
\hline $5900 \ldots$ & 4.00 & 3.53 & 5.59 & 3.64 & 7.87 & 3.74 \\
\hline \multicolumn{7}{|c|}{$P_{0}=10.0$ days } \\
\hline 5100. & 4.78 & 3.37 & 5.49 & 3.41 & 6.71 & 3.47 \\
\hline $5300 \ldots \ldots \ldots$ & 4.76 & 3.44 & 5.81 & 3.50 & 7.11 & 3.56 \\
\hline $5500 \ldots \ldots \ldots$ & 4.78 & 3.50 & 6.12 & 3.58 & 7.77 & 3.65 \\
\hline $5700 .$. & 4.63 & 3.55 & 6.13 & 3.64 & 8.15 & 3.72 \\
\hline 5900. & 3.79 & 3.54 & 6.10 & 3.69 & 8.56 & 3.80 \\
\hline \multicolumn{7}{|c|}{$P_{0}=10.5$ days } \\
\hline 5100. & 5.05 & 3.41 & 5.86 & 3.46 & 7.18 & 3.52 \\
\hline $5300 \ldots$ & 5.05 & 3.48 & 6.20 & 3.54 & 7.65 & 3.61 \\
\hline $5500 \ldots \ldots \ldots$ & 5.10 & 3.55 & 6.58 & 3.63 & 8.32 & 3.70 \\
\hline $5700 \ldots \ldots \ldots$ & 4.94 & 3.60 & 6.62 & 3.69 & 8.79 & 3.77 \\
\hline $5900 \ldots \ldots \ldots$ & 4.06 & 3.59 & 6.63 & 3.74 & 9.26 & 3.85 \\
\hline
\end{tabular}

Pel (1976). Temperatures were calculated for each star-for the Moffett-Barnes data using $B-V$ colors and the temperature scale of Teays \& Schmidt (1987); and for the Pel data, using the Pel temperature scale based on Walraven colors. Mean temperatures for the period range between 9 and 11 days were slightly under $5500 \mathrm{~K}$ for the Moffett-Barnes Cepheids (nine stars) and slightly over $5600 \mathrm{~K}$ for the Pel Cepheids (11 stars). Given these results we shall take $5500 \mathrm{~K}$ as our estimate for the temperature of a galactic Cepheid with a period near 10 days.

However, even if one considers a restricted range around $5500 \mathrm{~K}$, say $\pm 200 \mathrm{~K}$, the multiplicity of entries in Tables 1 and 2 does not sufficiently constrain the mass and luminosity of Cepheids at the resonance center. Later in this work, we shall combine our bump Cepheid calculations with models for the beat Cepheids, in an attempt to strengthen these constraints.

\subsection{Cepheids in the Magellanic Clouds}

Andreasen \& Petersen (1987) and Andreasen (1988) have performed Fourier decompositions on photographic observations of Cepheids in the Large Magellanic Cloud (LMC) and Small Magellanic Cloud (SMC), respectively. Though the data are noisy, a comparison with the Galactic Cepheids hints strongly at similar resonance progressions but with the center occurring at a slightly longer period-perhaps near 11 days in both the $\operatorname{LMC}(Z \cong 0.008)$ and $\operatorname{SMC}(Z \cong 0.005)$ as opposed to 10 days for the Galaxy.

Accordingly, we present in Table 3 the results of LNA calculations for lower metallicities $(Z=0.01,0.004$, and 0.002$)$ and with an assumed resonance center $P_{2} / P_{0}=11$ days. These models were constructed with OPAL opacities, but we would expect very similar results with OP opacities, given that the two opacity calculations yield masses and luminosities whose differences narrow with decreasing metallicity and indeed have already nearly converged at $Z=0.01$ (compare Tables 1 and 2).

The numbers in Tables 1-3 are consistent with Cepheid masses and, particularly, luminosities that differ little between the Galaxy and the Magellanic Clouds. However, if the Galactic metallicity verges toward $Z=0.03$ (Stothers \& Chin 1991), then our results imply that Galactic Cepheids with $P_{0} \sim 10$ days are more massive than their counterparts in the LMC and SMC by perhaps $1 M_{\odot}$ or even more. In a subsequent section we shall argue against a high Galactic metallicity, based upon a comparison of beat and bump Cepheid periods.

\section{THE BEAT CEPHEIDS}

Table 4 lists fundamental periods, $P_{0}$, and period ratios, $P_{1} / P_{0}$, for 12 Galactic double mode Cepheids, 11 from Balona (1985), and the remaining star, EW Scuti, from Cuypers (1985). We attempted to model these Cepheids, again using the LNA

TABLE 3

Pulsation MOdels With $P_{2} / P_{0}=0.5$ For $P_{0}=11.0$ Days (OPACITY Is OPAL)

\begin{tabular}{ccccccccc}
\hline \hline & \multicolumn{2}{c}{$Z=0.01$} & & \multicolumn{2}{c}{$Z=0.004$} & & \multicolumn{2}{c}{$Z=0.002$} \\
\cline { 2 - 3 }$T_{e}$ & $M$ & $\log L$ & & $M$ & $\log L$ & & $M$ & $\log L$ \\
\hline $5100 \ldots \ldots \ldots$ & 5.29 & 3.46 & & 5.16 & 3.45 & & 5.22 & 3.45 \\
$5300 \ldots \ldots \ldots$ & 5.34 & 3.53 & & 4.98 & 3.50 & & 4.93 & 3.50 \\
$5500 \ldots \ldots \ldots$ & 5.39 & 3.59 & & 4.83 & 3.56 & & 4.65 & 3.55 \\
$5700 \ldots \ldots \ldots$ & 5.35 & 3.65 & & 4.66 & 3.61 & & 4.42 & 3.60 \\
$5900 \ldots \ldots \ldots$ & 5.08 & 3.69 & & 4.23 & 3.64 & & 3.97 & 3.62 \\
\hline
\end{tabular}


TABLE 4

DOUble-Mode CePHeidS

\begin{tabular}{|c|c|c|c|}
\hline Star Number & Star Name & $P_{0}$ & $P_{1} / P_{0}$ \\
\hline & TU Cas & 2.1393 & 0.7097 \\
\hline & U T & & 105 \\
\hline 3. & VX Pup & 3.0109 & 0.710 \\
\hline & AP Vel & 3.1278 & 0.703 \\
\hline & BK Cen & 3.1739 & 0.700 \\
\hline 6. & & 3.3344 & 0.706 \\
\hline & Y Car & 6398 & 0.703 \\
\hline 8. & AX Vel & 3.6 & 0.705 \\
\hline & GZ Car & 4.1 & 0.7 \\
\hline 10. & BQ Ser & 4.2707 & 0.705 \\
\hline 11. & EW Sct & 5.8195 & 0.698 \\
\hline 12. & V367 Sct & 6.2931 & 0.69 \\
\hline
\end{tabular}

code of Aikawa \& Simon (1983). This project proved more difficult than that for the bump Cepheids described above. In particular, it was not possible to specify a temperature and then uniquely infer values for $M$ and $\log L$. Rather, we proceeded as follows. For each of the stars in Table 4, we first chose a metallicity, then specified a mass, guessing at the values of the pair $\left(\log L, \log T_{e}\right)$ and iterating until we found values of $\log L$ and $\log T_{e}$ which produced periods matching the observed ones. Subsequently, another mass was specified and another pair $\left(\log L, \log T_{e}\right)$ inferred, and so on, until we had covered the reasonable mass range for the star in question. Then, we chose different metallicities, and repeated the procedure, once again obtaining a value of luminosity and temperature for each metallicity and mass.

The results are presented in Figures 1-12, one figure for each star, numbered as in Table 4. Each point in the plots represents a model which reproduces the observed periods. The luminosity and mass of the model are given along the ordinate and abscissa, respectively, while the temperature $\left(T_{e} / 100\right)$ is written in next to each point. The differing symbols stand for different assumed metallicities as follows: $Z=0.03$ (open squares); $Z=0.02$ (filled squares); $Z=0.015$ (open triangles); $Z=0.01$ (filled triangles). All of the models were calculated using OPAL opacities.

A quick perusal of Figures 1-12 indicates that the points themselves do little to fix the parameters of the double-mode Cepheids. For example, in Figure 1, while we might reasonably eliminate the higher temperature models (because they lie beyond any reasonable blue edge), this still leaves a wide array of possible masses, luminosities and metallicities. However, it is possible to make some progress here, using the results of the previous section on bump Cepheids.

Let us begin with the generic equation for evolutionary models crossing the instability strip:

$$
\log L=4.0 \log M+b,
$$

where the slope $s=4.0$ is that suggested by Stothers \& Chin (1991) for models calculated with OPAL opacities. To determine possible values for the intercept $b$ we shall use the bump Cepheid results along with a circumstance reported in $\S 2.2$, namely that the $P_{2} / P_{0}=0.5$ resonance appears to be located near 11 days in the LMC and SMC. This hints at a metallicity dependence wherein the resonance center occurs at longer periods as $Z$ is decreased.

With this in mind, let us specify $P_{\mathrm{RC}}=10.5,10.0$, and 9.5 days for $Z=0.01,0.02$, and 0.03 , respectively. Then, adopting the fiducial temperature $T_{e}=5500 \mathrm{~K}$ (see above), we obtain from Table 1 , the respective pairs $(M, \log L)=(5.07,3.55)$,

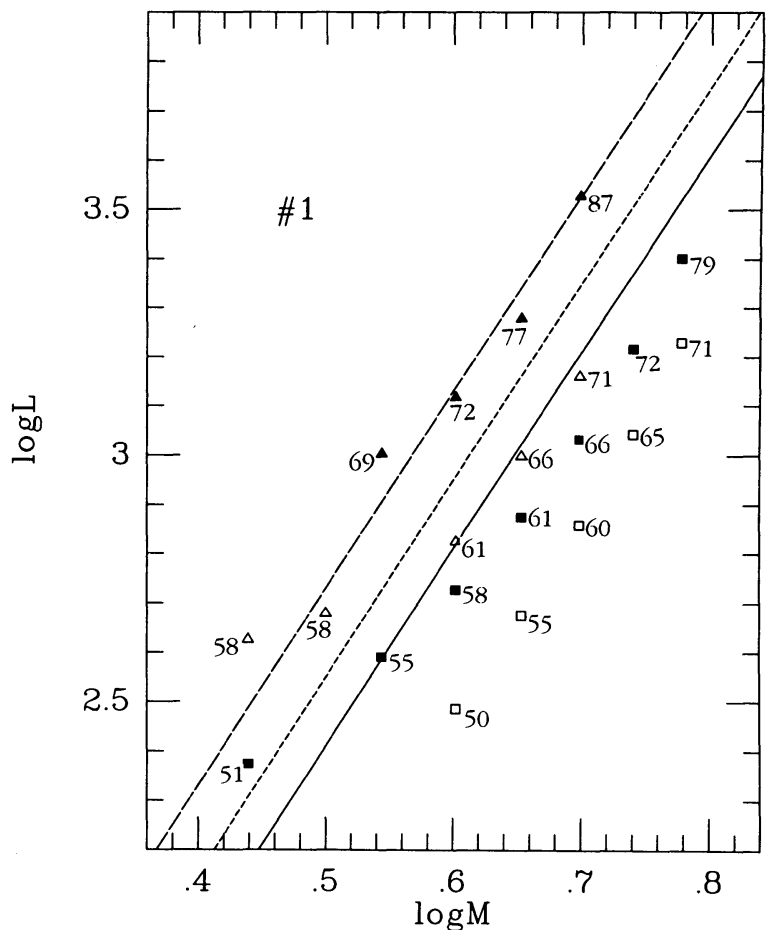

FIG. 1.-Linear pulsation models (with OPAL opacities) for beat Cepheid 1 (see Table 4). Each symbol represents a model which matches the observed periods $P_{0}$ and $P_{1}$ : (open squares) $Z=0.03$; (filled squares) $Z=0.02$; (open triangles) $Z=0.015$; (filled triangles) $Z=0.01$. Luminosities and masses of models are given along ordinate and abscissa, respectively; temperatures $\left(T_{e} / 100\right)$ are written in next to each point. The straight lines are evolutionary loci according to eq. (1) with the intercept determined by modeling the bump Cepheid resonance (see text): (long-dashed line) $Z=0.01$; (small-dashed line) $Z=0.02 ;$ (solid line) $Z=0.03$.

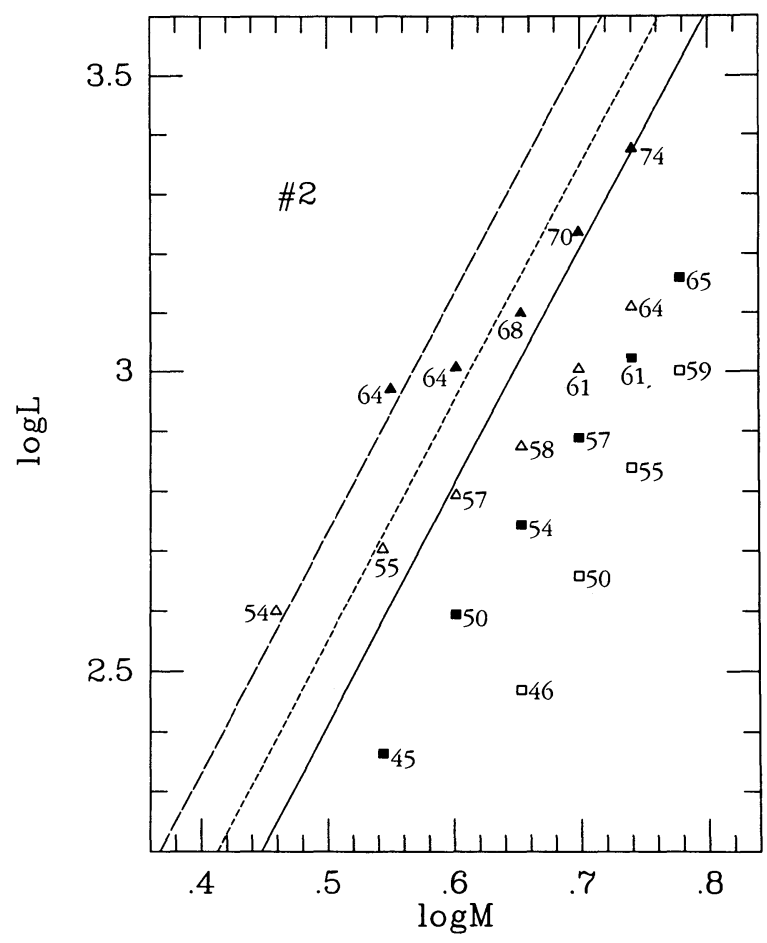

Fig. 2.-Same as Fig. 1, but for star 2 


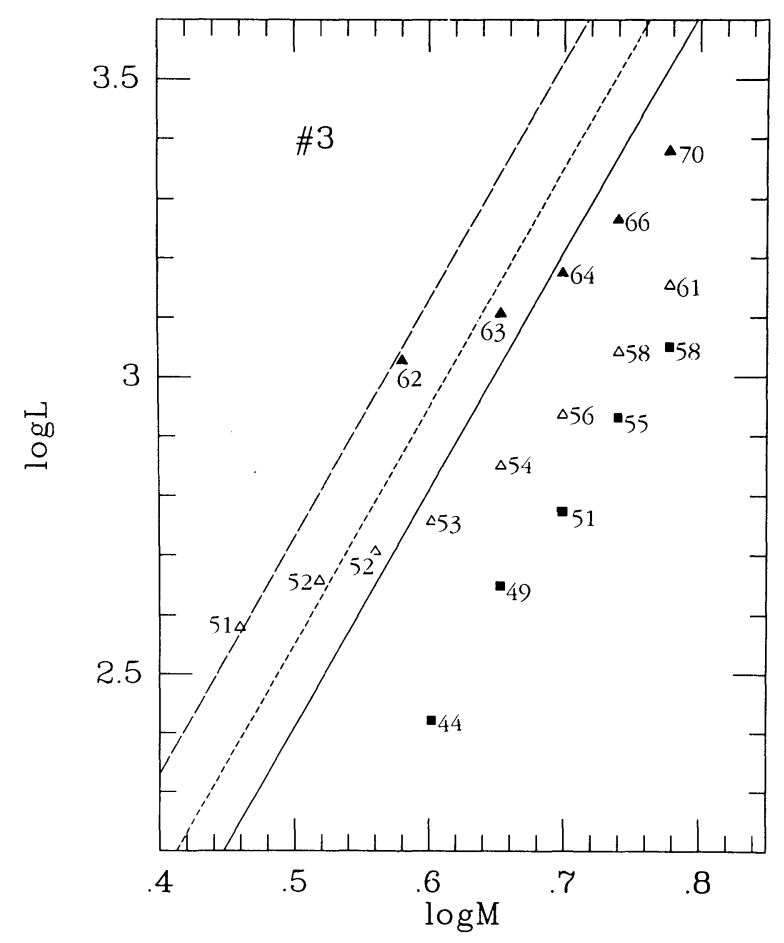

Fig. 3.-Same as Fig. 1, but for star 3

$(5.62,3.55)$ and $(6.08,3.55)$ at the resonance center for $Z=0.01$, 0.02 , and 0.03 . Use of each of these pairs in equation (1) yields the relations

$$
\begin{array}{ll}
\log L=4.0 \log M+0.73 & (Z=0.01) \\
\log L=4.0 \log M+0.55 & (Z=0.02) \\
\log L=4.0 \log M+0.41 & (Z=0.03)
\end{array}
$$

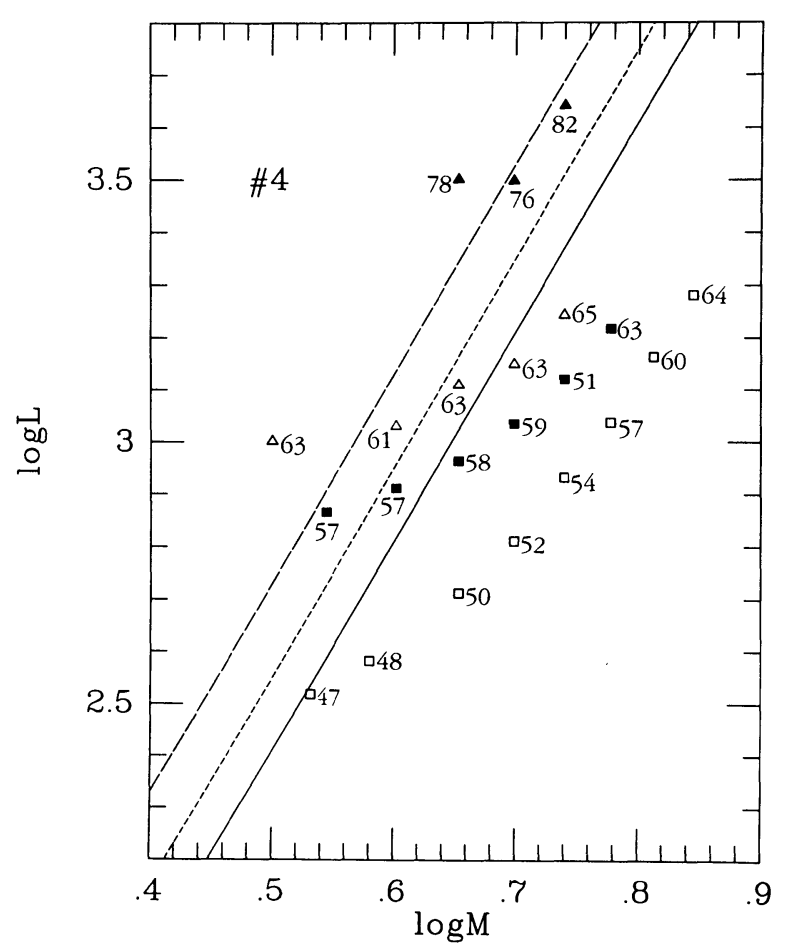

Fig. 4.-Same as Fig. 1, but for star 4

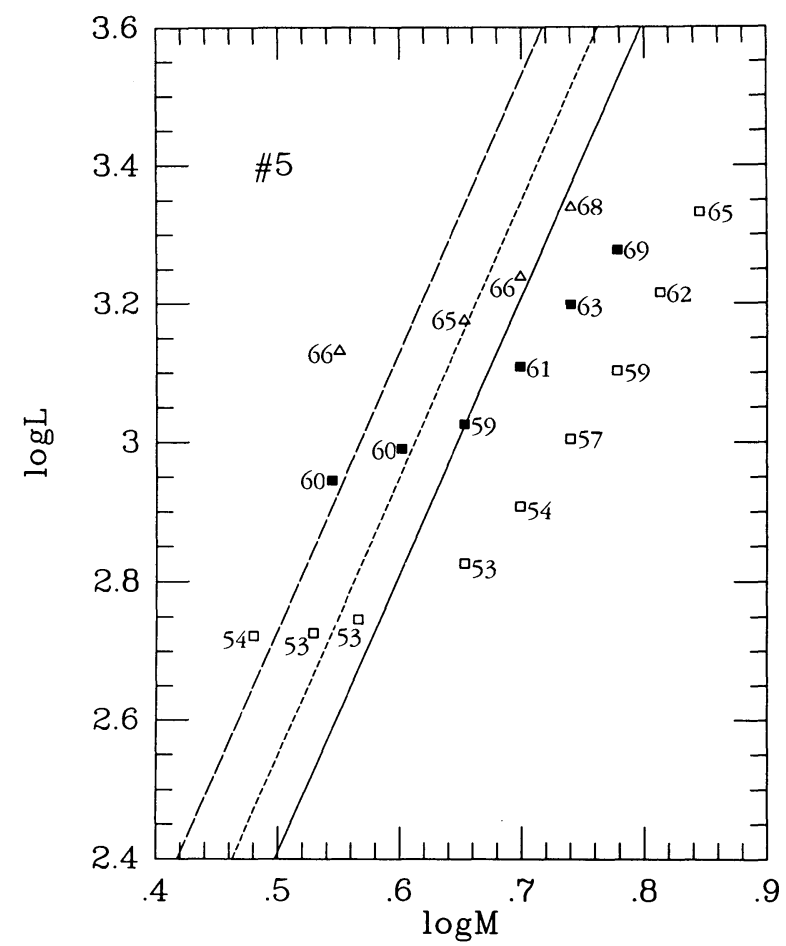

FIG. 5.-Same as Fig. 1, but for star 5

Although these equations result from specific choices in Table 1 , we shall argue later that the conclusions we are about to draw would be little changed by virtually any choice of points in Table 1.

The loci represented by equations (2), (3), and (4) have been rendered on Figs. 1-12 as dashed, small dashed, and solid lines, respectively. The first of these implies that Galactic Cepheids

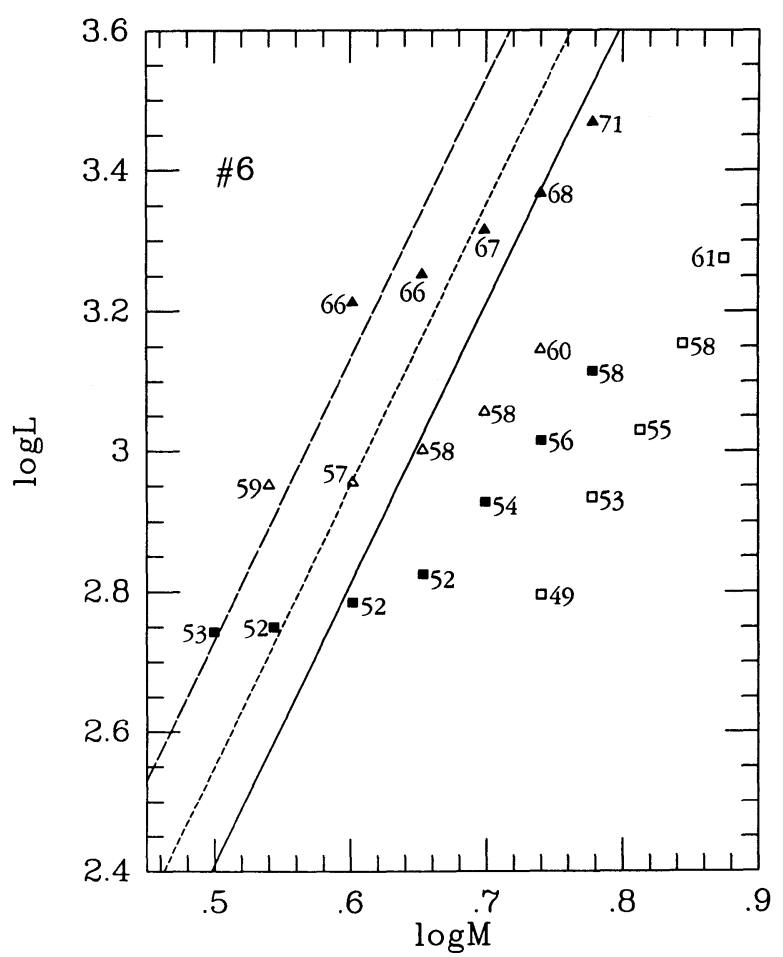

Fig. 6.-Same as Fig. 1, but for star 6 


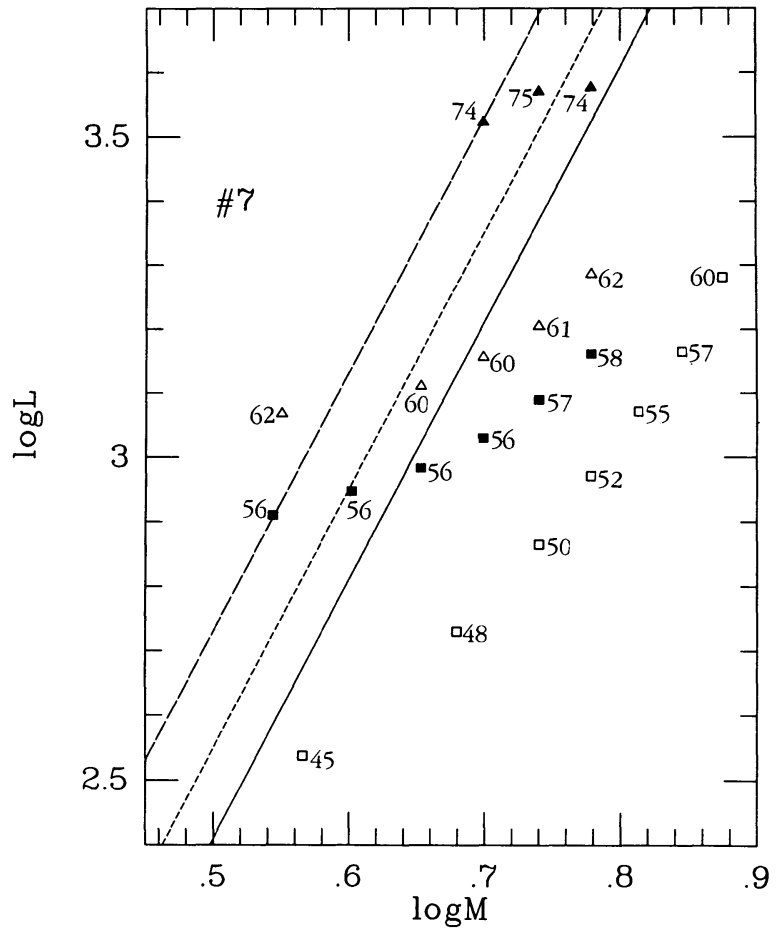

FIG. 7.-Same as Fig. 1, but for star 7

with periods around 10 days have on the average a metallicity $Z=0.01$, the second that their metallicity is $Z=0.02$, and the last a metallicity $Z=0.03$. Let us now ask, what would constitute a reasonable temperature range for the beat Cepheids? If the bump Cepheid temperature at resonance center, $P_{0} \cong 10$ days, is $T_{e} \cong 5500 \mathrm{~K}$, then the beat Cepheids, $2 \lesssim P_{0} \lesssim 6$ days,

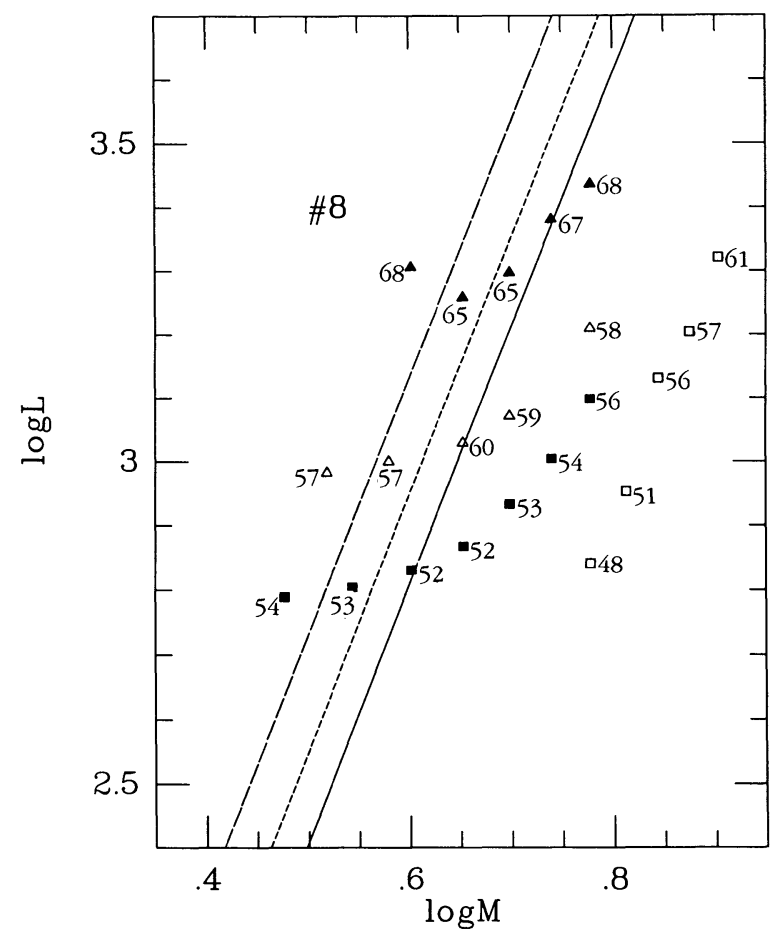

Fig. 8. - Same as Fig. 1, but for star 8

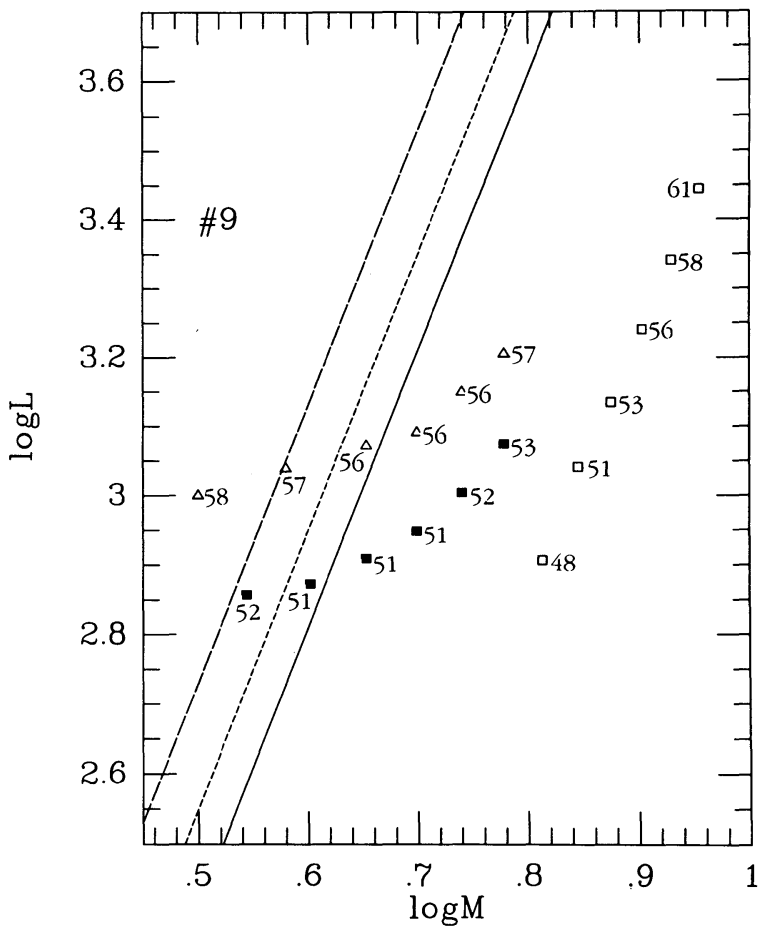

Fig. 9.-Same as Fig. 1, but for star 9

generally ought to have higher temperatures, due to the wellknown slope of the instability strip in the H-R diagram and the properties of the period-luminosity relation. Thus let us estimate for the beat Cepheids the range $6300 \mathrm{~K} \lesssim T_{e} \lesssim 5700 \mathrm{~K}$. This range is a bit more generous than that determined by Balona \& Stobie (1979) for a subset of the double-mode stars.

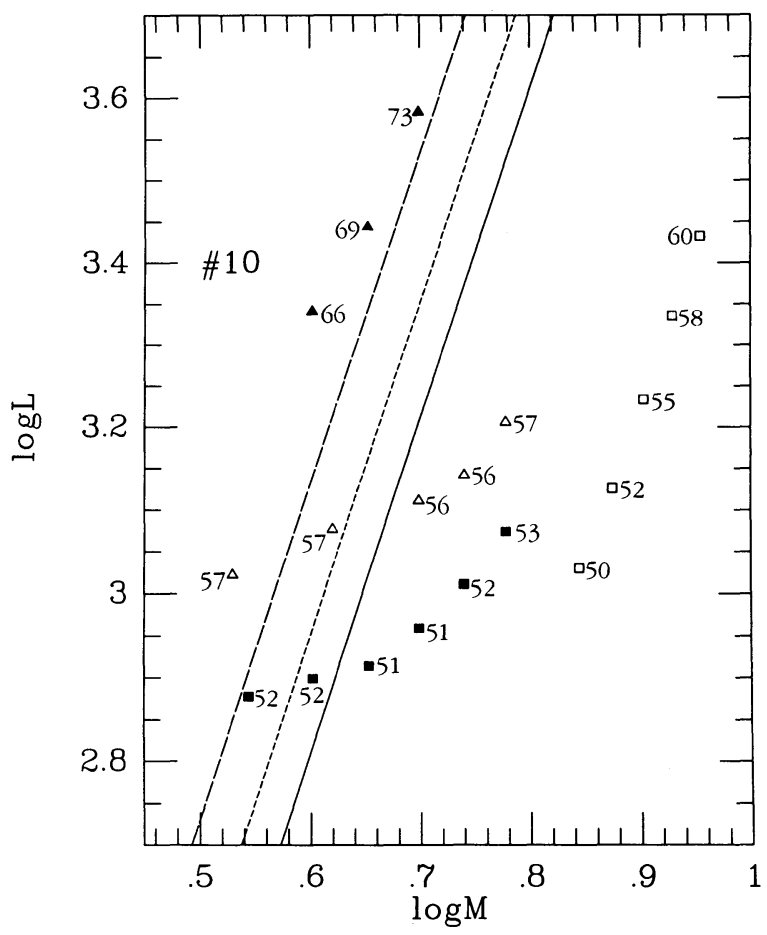

FIG. 10.-Same as Fig. 1, but for star 10 


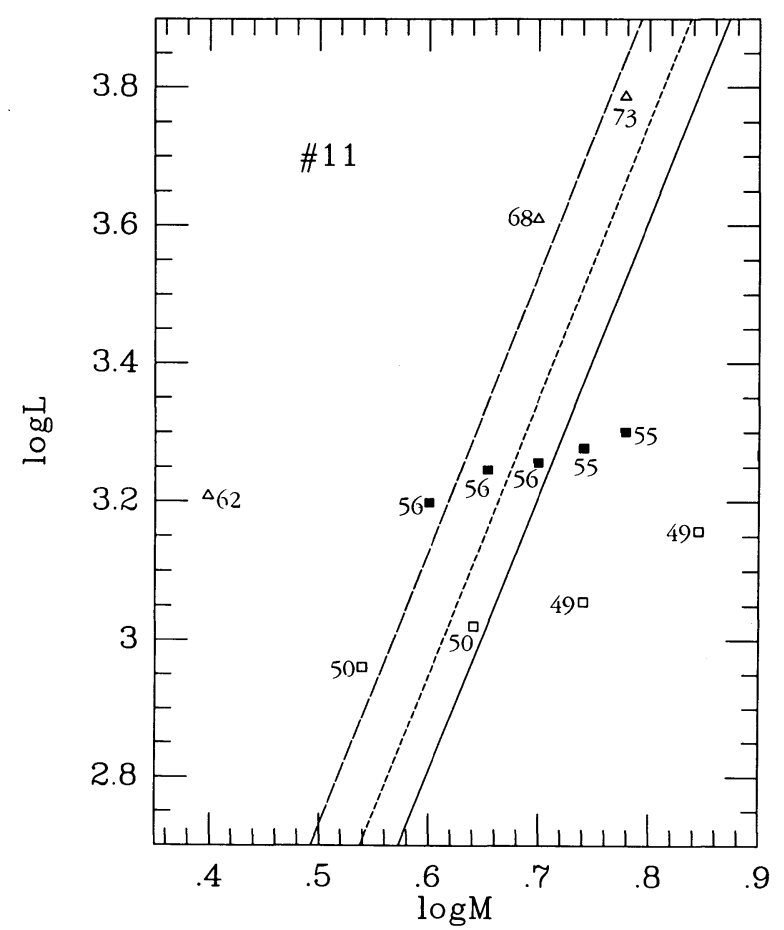

FIG. 11.-Same as Fig. 1, but for star 11

Now, let us choose one of the diagrams, e.g., Figure 2, and, for simplicity, first assume that all Galactic Cepheids have the same metallicity, say $Z=0.03$. Then, consistency requires that the solid line (the $Z=0.03$ evolutionary locus) pass through one of the open squares (models that reproduce the observed beat periods for $Z=0.03$ ) at a reasonable temperature. It is easy to see that this condition is not satisfied in Figure 2. The

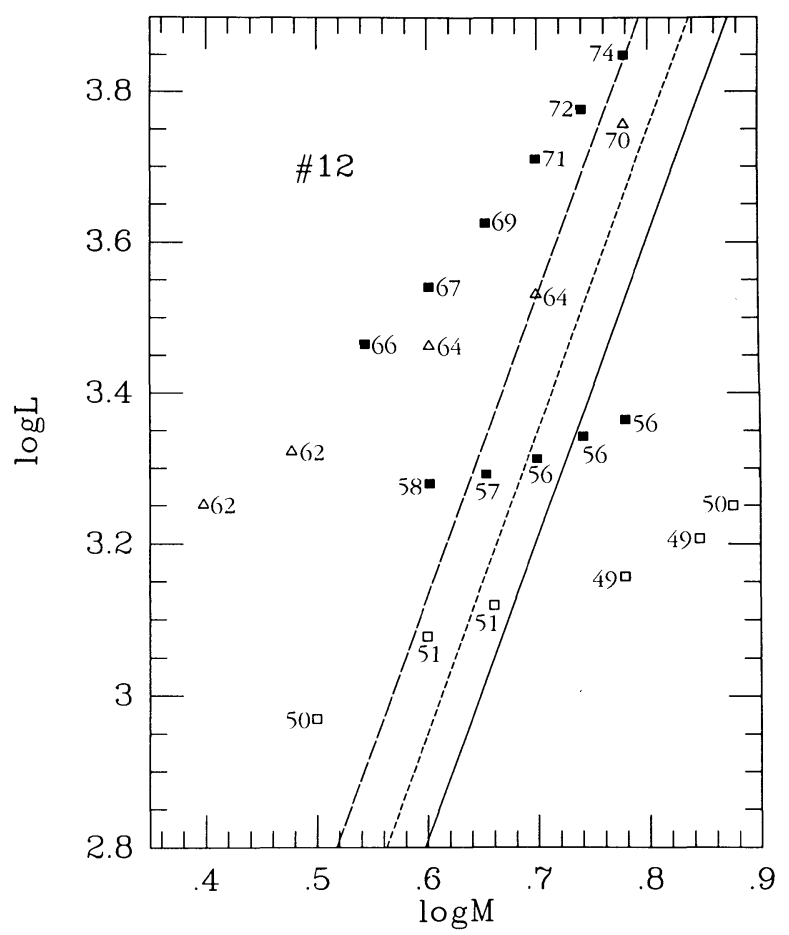

Fig. 12.-Same as Fig. 1, but for star 12 solid line lies well above the locus of the beat models: that is to say, at $Z=0.03$, evolutionary models which reproduce the bump Cepheid resonance are too luminous at given mass to yield the observed beat periods. This is the case for most of the double-mode stars, in particular stars $1,2,3,6,8,9$, and 10. For four other beat Cepheids (stars 4, 7,11, and 12) a solution may be found at $Z=0.03$, but only for $T_{e} \lesssim 5000 \mathrm{~K}$, which, according to our earlier arguments, is much too cool. Only in the case of star 5 does there exist a halfway reasonable solution at $Z=0.03$, but even here models of lower metallicity yield results which are more satisfactory.

To continue, suppose we now take all of the Galactic Cepheids to have $Z=0.02$. According to Figures $1-12$, this choice is clearly better. In a number of cases, we attain the consistency that was sought. For example, consider star 4 (Fig. 4). Here the small-dashed line $(Z=0.02$ evolutionary locus) intersects the filled squares $(Z=0.02$ beat Cepheid locus), yielding a reasonable and consistent solution with $M \cong 4 M_{\odot}$, $\log L \cong 2.9, T_{e} \cong 5700 \mathrm{~K}$. Similar values are obtained for stars 5 and 7 , while for stars 11 and 12 , we get $T_{e} \cong 5600 \mathrm{~K}, M \cong 5$ $M_{\odot}, \log L \cong 3.3$.

However, not all the double-mode stars can be accommodated at $Z=0.02$. Consider, once again, the case portrayed in Figure 2. Here the small-dashed line lies well above the filled squares, indicating that a lower metallicity is required. In fact, the data in this figure point to a solution with $0.010 \leq Z \leq 0.015$, and $M \cong 3.5 M_{\odot}, \log L \cong 2.9$. Other stars which seem to indicate metallicities $\lesssim 0.015$ are those portrayed in Figures 1, 3, 6, 8, 9, and 10. It should be emphasized that for none of the dozen double-mode Cepheids is a metallicity $Z>0.02$ required.

\section{A CRUDE PORTRAIT OF THE GALACTIC CEPHEIDS}

The large number of LNA models constructed for the present study are consistent with the following picture of the Galactic classical Cepheids. These stars exhibit a considerable range of metallicity, perhaps spanning an interval as large as: 0.01 (e.g., star 2 ) $\lesssim Z \lesssim 0.02$ (e.g., star 5). So far as the pulsation results are concerned there seems no reason to assume that any of the beat or bump Cepheids are more metal-rich than $Z=0.02$, and, if the present models are accurate, a high metallicity is precluded for many of the stars. The range in metallicity gives rise to a range in location of the bump Cepheid resonance, whose width in period is $\lesssim 1$ day, with the condition $P_{2} / P_{0}=0.5$ occurring at longer period for lower metallicity. This implies that there must also be a real spread of $\lesssim 1$ day in the Fourier diagrams for galactic Cepheids (Simon \& Moffett 1985). Thus the resonance center may not be precisely defined, as opposed to merely not being precisely measured due to observational uncertainty.

How do the pulsation results compare with those from published evolutionary tracks? Tables 1 and 2 along with Figures 1-12 point to masses somewhat less than $4 M_{\odot}$ at periods of 2 or 3 days, ranging up to 5 or $6 M_{\odot}$ at 10 days. Over this same range the luminosities go from $\log L \lesssim 3.0$ to $\log L \gtrsim 3.5$. To these parameters we may compare the evolutionary tracks of Schaller et al. (1992) and Schaerer et al. (1993, hereafter the Geneva Group), and Stothers \& Chin (1991), all calculated with OPAL opacities. The models of the Geneva Group include modest convective core overshoot $\left(d / H_{p}=0.2\right)$ while the Stothers-Chin models are constructed without overshoot.

At $Z=0.02$, the Schaller et al. models of 4 and $5 M_{\odot}$ display 
stunted blue loops at luminosities of about $\log L=2.7$ and $\log$ $L=3.2$, respectively. While these stars are dimmer than those suggested by pulsation analysis, a worse problem is that the blue loops fail to penetrate anywhere near a temperature $T_{e}=5500 \mathrm{~K}$. At $Z=0.03$ and $M=5 M_{\odot}$, the Stothers-Chin luminosities are also low, even if one brightens them by $\Delta \log$ $L=0.12$ to extrapolate to $Z=0.02$. Once more, the blue loops fall far short of $5500 \mathrm{~K}$, a result which, according to the authors' report, is also true for the $Z=0.02$ models.

The Schaerer et al. calculations at $Z=0.008$ appear more promising. Here the blue loops for 4 and $5 M_{\odot}$ penetrate to or beyond $5500 \mathrm{~K}$, sweeping through a domain on the H-R diagram which corresponds to a "slow phase of nuclear burning." The luminosities are also higher here, with the models attaining brightnesses close to those suggested by the beat and bump Cepheids. We shall have more to say about this comparison in a later section.

\section{UNCERTAINTIES}

As mentioned above, the beat Cepheid period ratios have proven complicated to model in that the solutions are not always crisp and well-defined. We estimate that an average point in Figures 1-12 carries random errors of \pm 0.05 in $\log L$, and $\pm 100 \mathrm{~K}$ in $T_{e}$. These uncertainties involve only the choice of a convergence criterion for the Newton-Raphson iteration, not the physical input to the models, which could potentially cause systematic errors and shall be treated below. Of course, the beat Cepheid loci in Figures 1-12 are generally quite regular and have considerably less uncertainty than do the individual points. In addition, let us mention that we have tested the sensitivity of our calculations to zoning in the LNA code and find that the results vary little as one goes from about 70 to 400 zones.

Figure 12 portrays some of the problems in the beat modeling. The loci for $Z=0.03$ and $Z=0.015$ (open squares and triangles, respectively) here have irregular shapes which may or may not be due to random uncertainties in individual points. Worse still, we note that the sequence for $Z=0.02$ is doublevalued! In addition to the "normal" locus, there exists another at considerably higher luminosity. We have found such double sequences in all of the longer period stars (numbers 7 to 12), always for $Z=0.02$ or 0.015 . However, in all cases, the temperatures associated with the brighter loci are so hot that the points lie beyond any reasonable blue edge and thus cannot be considered as solutions. It should also be emphasized that, although we have searched carefully, we could not find a higher luminosity sequence for any star at $Z=0.03$. Finally, we note that, to avoid clutter, we have plotted the higher loci in Figure 12 only. This case is typical, and adequately demonstrates the phenomenon.

Turning to the bump Cepheid solutions in Tables 1-3, we find here a much sharper convergence than with beat models, and hence a considerably smaller random uncertainty. This means that the individual straight lines drawn in Figures 1-12 are quite precise. However, one still needs to ask what would be the effect of changing the intercepts of these lines by choosing different points in Tables 1 and 2 to plug into equation (1). The answer is that such an exercise would produce little change in the qualitative conclusions drawn in $\S 4$. In particular, for $Z=0.03$, even the extreme choice, $P_{\mathrm{RC}}=10.5$ days, $T_{e}=5900 \mathrm{~K}$, which yields the point, $M=7.78, \log L=3.80$, does not suffice to bring the evolutionary loci (solid lines) down to meet the open squares (beat models) in Figures 1-3, 6, and
8-11. The $Z=0.02$ lines are affected even less. Only for $Z=0.01$ would another extreme choice, $P_{\mathrm{RC}}=9.5$ days, $T_{e}=$ $5900 \mathrm{~K}$ have an important effect, implying substantially lower masses for the shorter period stars. However, there is no reason to believe that such a choice is correct, and, particularly, that such a high temperature characterizes the resonance center. Finally, we note that changing the slope in equation (1) to a value which appeared in the earlier literature-namely, $s=3.6$-would also have little effect on Figures 1-12.

Let us move then to the question of possible systematic errors in the calculated period ratios. To get at this we shall examine a known uncertainty in the calculations, namely the opacity difference between OPAL and OP. Figures 13, 14, and 15 treat the data for typical stars 2,5 , and 11 , respectively, but using OP opacities to calculate the beat Cepheid model points, and instead of Table 1 , the corresponding entries in Table 2 (i.e., $T_{e}=5500 \mathrm{~K}$, and $P_{\mathrm{RC}}=9.5,10.0$ and 10.5 days for $Z=0.03,0.02$, and 0.01 , respectively) to form the straight lines in the diagrams, as described above in $\S 3$.

Comparing Figures 13, 14, and 15 with the corresponding OPAL diagrams in Figures 2, 5, and 11, respectively, we find only small differences. Slightly higher masses and lower luminosities are implied by OP. This is despite the fact that the OP beat Cepheid period ratios $\left(P_{1} / P_{0}\right)$ are systematically smaller than those calculated with OPAL by a reasonably large amount, of the order 0.005 (Kanbur \& Simon 1994). The reason for the small effect is, of course, that the bump Cepheid period ratios $\left(P_{2} / P_{0}\right)$ are also lower with OP, so that both the lines and the points are translated downward in the diagrams, their loci intersecting (and thus yielding solutions) at metallicities very similar to those implied with OPAL. It is clear from these examples that if one wants to alter qualitatively the conclusions reached above in $\S 4$, a differential effect must be invoked, wherein the $P_{2} / P_{0}$ and $P_{1} / P_{0}$ period ratios change differently.

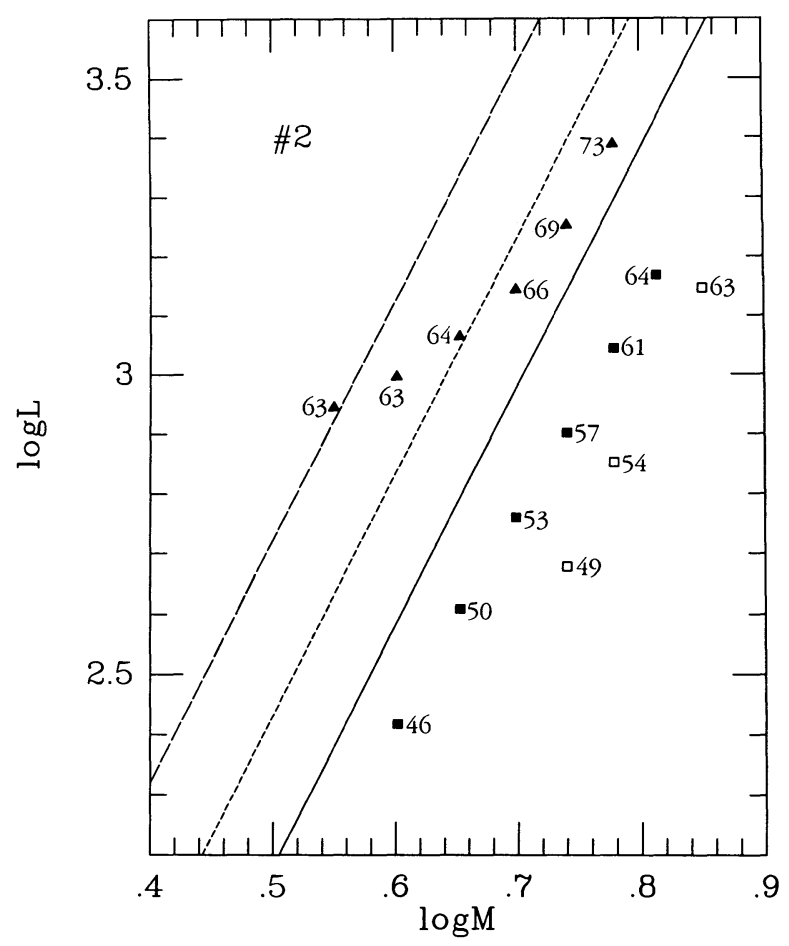

Fig. 13.-Same as Fig. 2, but calculations use OP opacities 


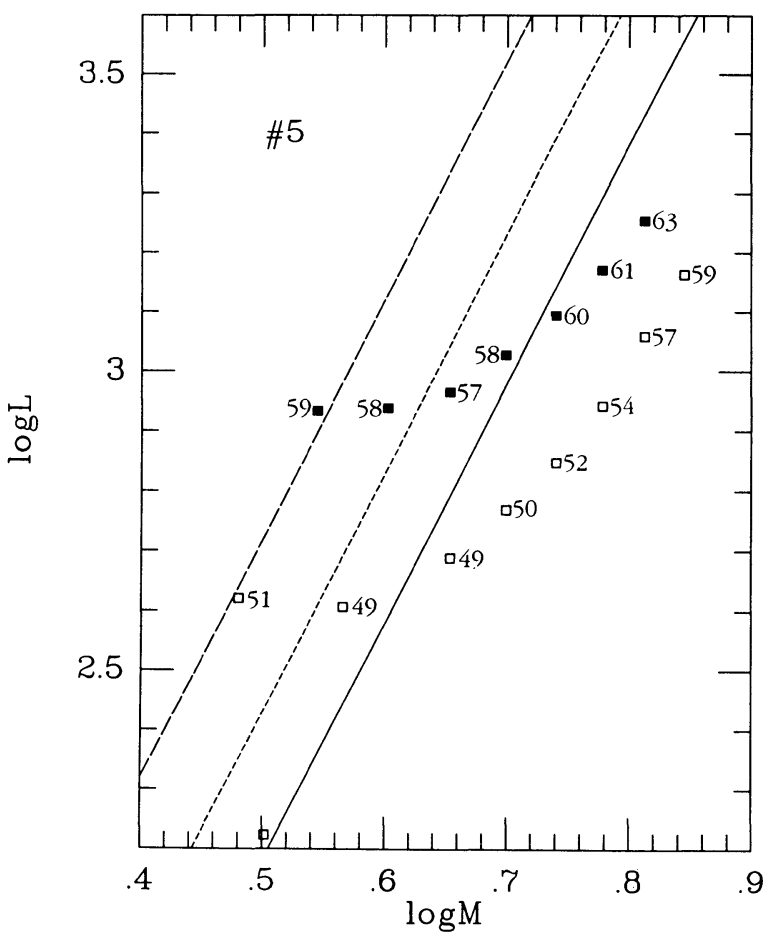

Fig. 14.--Same as Fig. 5, but calculations use OP opacities

\section{SUMMARY OF RESULTS}

Let us recapitulate our procedure for treating the galactic Cepheids. We assume a slope $s=4.0$ in the evolutionary luminosity-mass relation (eq. [1]) and, for given metallicity, fix the intercept by modeling the bump Cepheid resonance, $P_{2} / P_{0}=0.5$. Next, we calculate models for each of the dozen

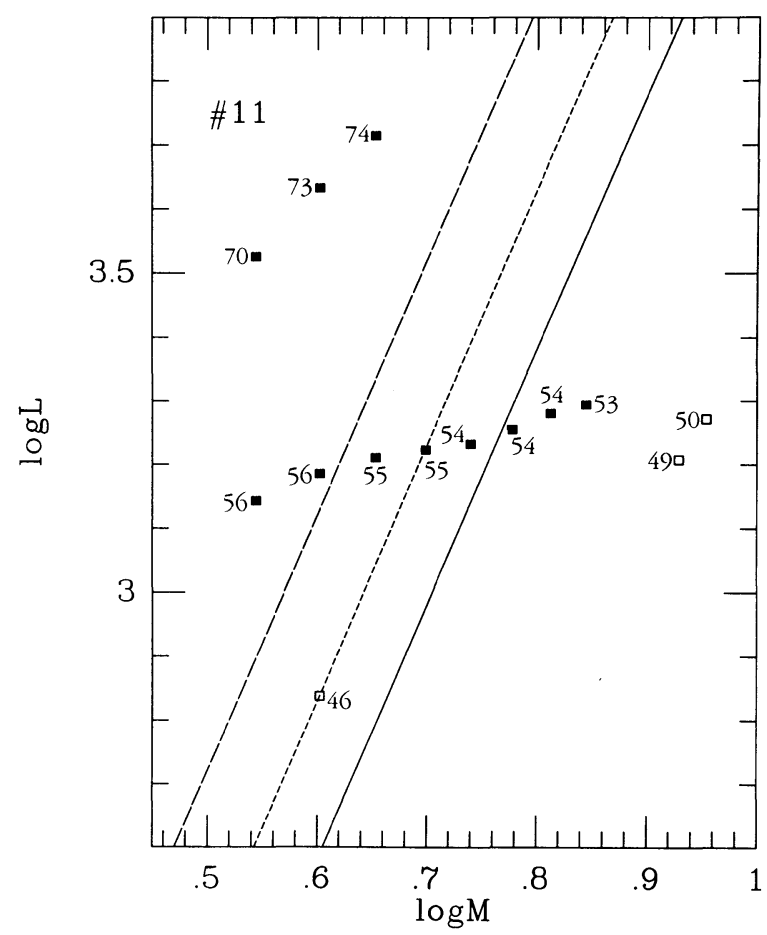

FIg. 15.-Same as Fig. 11, but calculations use OP opacities known beat Cepheids and require that their masses and luminosities agree with the evolutionary relationship, always for a reasonable temperature.

From the above exercise, we have concluded that (1) the masses and luminosities of Galactic classical Cepheids range from $M \lesssim 4 M_{\odot}, \log L \lesssim 3.0$ at $P \cong 3$ days, to $M \lesssim 6 M_{\odot}$, $\log L \gtrsim 3.5$ at 10 days; ${ }^{2}$ and that (2) these stars span a range of metallicity, perhaps as large as $0.01 \lesssim Z \lesssim 0.02$, with average value $Z \cong 0.015$. No evidence supports the existence of beat or bump Cepheids with $Z>0.02$.

Expanding our analysis to the Magellanic Clouds, we find that, if, as the data hint, the bump Cepheid resonance occurs at $P \gtrsim 11$ days in the LMC and SMC, then the masses and (particularly) the luminosities of bump Cepheids in the Clouds are very similar to those of their counterparts in the Galaxy.

The above conclusions could be upset if (1) the evolutionary luminosity-mass relation for Cepheids with $2 \lesssim P \lesssim 10$ days really cannot be characterized by a simple linear formulation such as that in equation (1); or (2) the insertion of complete and correct physics in the LNA codes would systematically alter the calculated period ratios by a substantial amount (say, $\left.\left|\Delta\left(P / P_{0}\right)\right|>0.005\right)$ and in a manner that acts differently for $P_{1} / P_{0}$ and $P_{2} / P_{0}$.

\section{FUTURE WORK}

A number of areas could profitably be investigated further in connection with the current results. We describe possible future work briefly in what follows.

1. Stellar evolution.-As shown above in $\S 4$, the pulsation results indicate that the evolutionary models may be somewhat underluminous, particularly at higher metallicity. This could perhaps be remedied by a modest increase in the amount of convective core overshoot. The problem of stunted blue loops for models with $4 \lesssim M \lesssim 5 M_{\odot}$ is perhaps more serious. This difficulty has been discussed by Stothers \& Chin (1993) and a possible solution proposed in terms of an opacity increase (Stothers \& Chin 1994). However, it remains to be seen whether evolutionary models can be constructed which reproduce the beat and bump Cepheid period ratios at reasonable temperatures and during "slow" nuclear phases. Future evolution calculations ought to aim at specifically mimicking each of 12 beat Cepheids as well as the bump Cepheid resonance near 10 days. The data points in Figures $1-15$ and Tables $1-3$ could serve as a guide for such calculations. If, in the end, a discrepancy remains between evolution and pulsation results, its nature may well suggest how either or both might be modified.

2. The Magellanic Clouds.-The assignment of a period near 11 days to the bump Cepheid resonance in the Magellanic Clouds is based upon extremely noisy (and ultimately inadequate) data. Light curves more suitable for Fourier decomposition are needed for Cepheids in both the LMC and SMC. Such light curves, interpreted via pulsation models and compared with evolutionary tracks, could tell us much about how metallicity and Galactic history or morphology influence star formation. The necessary observations are well within current capabilities and ought to be performed and analyzed without delay.

3. Pulsation calculations.-A further set of LNA models

${ }^{2}$ We note that, at the low end of this range, our masses are in conflict with the lower limit, $M=5.9 \pm 0.4 M_{\odot}$, derived by Evans \& Bolton (1990) for the 3.8 day binary Cepheid, SU Cyg. However, despite the Evans-Bolton result, the masses of Cepheids remain controversial (see, e.g., Chiosi et al. 1992). 
ing to be constructed to test the effect on period ratios of I physical assumptions in the codes. An example of a physical mechanism which might well make a difference is convection, which was neglected in the current model calculations. In fact, Cox (1993) has indicated that a new iron convection zone (see, ${ }_{i l}$ also, Stothers \& Chin 1993), occasioned by the (higher) OPAL - opacities, reduces the $P_{2} / P_{0}$ period ratios in some models enough that the resonance condition $\left(P_{2} / P_{0}=0.5\right.$ at $P_{0}=10$ days) is attained for masses as high as 7 or $8 M_{\odot}$. However, this result is sensitive to the mixing length formulation for convection and, in particular, to the value chosen for the mixing length itself. While it would be of great interest to attempt to match observed beat and bump Cepheid parameters with models that include convection, such an undertaking is clearly beyond the scope of the current investigation and must be left to the future.

We are pleased to acknowledge support from the NASA Astrophysics Theory Program, under grant no. NAGW-2395. We are grateful to $M$. Seaton for furnishing opacity tables in advance of their publication.

\section{REFERENCES}

Aikawa, T., \& Simon, N. R. 1983, ApJ, 273, 346

Andreasen, G. K. 1988, A\&A, 191, 71

Andreasen, G. K., \& Petersen, J. O. 1987, A\&A, 180, 129

Balona, L. A. 1985, in Cepheids: Theory and Observation, ed. B. F. Madore (Cambridge: Cambridge Univ. Press), 17

Balona, L. A. \& Stobie, R. S. 1979, MNRAS, 189, 659

Chiosi, C. 1990, in Confrontation Between Stellar Pulsation and Evolution, ed. C. Cacciari \& G. Clementini (San Francisco: ASP), 158

Chiosi, C., Bertelli, G., \& Bressan, A. 1992, ARA\&A, 30, 235

Cox, A. N. 1993, in New Perspectives on Stellar Pulsations and Pulsating Variable Stars, ed. J. M. Nemec \& J. M. Matthews (Cambridge: Cambridge Univ. Press), 252

Cuypers, J. 1985, A\&A, 145, 283

Evans, N. R., \& Bolton, C. T. 1990, ApJ, 356, 630

Iglesias, C. A., \& Rogers, F. J. 1991, ApJ, 371, L73

Iglesias, C. A., Rogers, F. J., \& Wilson, B. 1992, ApJ, 397, 717
Kanbur, S. M. \& Simon, N. R. 1994, ApJ, 420,880

Kovács, G., \& Buchler, J. R. 1989, ApJ, 346, 898

Moffett, T. J., \& Barnes, T. G. 1985, ApJS, 58, 843

Moskalik, P., Buchler, J. R., \& Marom, A. 1992, ApJ, 385, 685

Pel, J. W. 1976, Ph.D. thesis, Univ. Leiden

Schaerer, D., Meynet, G., Maeder, A., \& Schaller, G. 1993, A\&AS, 98, 523 (Geneva Group)

Schaller, G., Schaerer, D., Meynet, G., \& Maeder, A. 1992, A\&AS, 96, 269

Seaton, M. J., Yu Yan, Mihalas, D., \& Pradhan, A. K. 1993, preprint

Simon, N. R., \& Moffett, T. J. 1985, PASP, 97, 1078

Simon, N. R. \& Schmidt, E. G. 1976, ApJ, 205, 162

Stothers, R. B., \& Chin, C.-w. 1991, ApJ, 381, L67 .1993, ApJ, 412, 294 1994, preprint

Teays, T. J. \& Schmidt, E. G. 1987, in Stellar Pulsation : a Memorial to John P. Cox, ed. A. N. Cox, W. M. Sparks, \& S. G. Starrfield (Berlin: Springer), 173 